\title{
Responsiveness of Oral Health-Related Quality of Life Questionnaires to Dental Caries Interventions: Systematic Review and Meta-Analysis
}

\author{
Nicole R. Aimée ${ }^{a} \quad$ Nailê Damé-Teixeira ${ }^{a} \quad$ Luana Severo Alves $^{b} \quad$ Gabriel Á. Borges $^{a}$

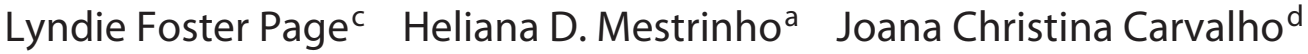 \\ ${ }^{a}$ Faculty of Health Sciences, University of Brasília (UnB), Brasília, Brazil; b School of Dentistry, Federal University of Santa \\ Maria (UFSM), Santa Maria, Brazil; ' Faculty of Dentistry, University of Otago (UO), Dunedin, New Zealand; d Faculty of \\ Medicine and Dentistry, Catholic University of Louvain (UCL), Brussels, Belgium
}

\section{Keywords}

Dental care · Surveys and questionnaires · Oral health .

Quality of life - Dental caries · Systematic review

\begin{abstract}
This systematic review and meta-analysis were undertaken to assess the responsiveness of validated oral health-related quality of life (OHRQoL) questionnaires to dental caries interventions in children, adolescents, and young adults. Studies eligible were randomized clinical trials (RCTs), controlled clinical trials (CCTs), and prospective case series (PCS), which had OHRQoL questionnaires answered before and after caries intervention(s). The main outcome was improvement in OHRQoL mean scores following caries intervention. Twentysix studies were selected for the quality assessment and 14 were selected for the meta-analysis. Most of the studies were PCS with a single group pretest and posttest study design $(n=19)$. Five studies were CCT and only 2 were RCT. The numbers of participants were 3,522 in the control group (baseline $=2,002$; final $=1,520$ ) and 5,917 in the test group (baseline $=3,102$; final $=2,815$ ). The age of the subjects ranged from 3 to 19 years. All studies showed significant im-
\end{abstract}

(c) 2019 S. Karger AG, Basel

\section{E-Mail karger@karger.com www.karger.com/cre \\ KARGER}

provement in OHRQoL following caries intervention. Most of nonrandomized studies $(n=15)$ had low or moderate risk of bias. The meta-analysis showed the effect of caries interventions (standardized weighted mean differences $=-1.24 ; 95 \%$ $\mathrm{Cl}:-1.68$ to $-0.81 ; p<0.001)$. However, high heterogeneity between the studies was found. The Grading of Recommendations Assessment, Development and Evaluation approach classified the quality of evidence as very low and its strength weak. In conclusion, there is evidence that the OHRQoL of children and adolescents improved following caries intervention procedures, but the quality of the evidence was very low. In spite of that, caries interventions are highly recommended as abstaining from treatment is likely to result in a deterioration of OHRQoL.

(c) 2019 S. Karger AG, Basel

\section{Introduction}

There is a growing understanding that oral health outcomes should be assessed not only by clinical findings but also by patients' reported outcomes through the application of structured and validated questionnaires 
[Tsakos et al., 2010; Antunes et al., 2013; Alves et al., 2012 Krisdapong et al., 2013; Turton et al., 2015; Aimée et al., 2017]. Validated questionnaires assessing oral health-related quality of life (OHRQoL) in children, adolescents, and young adults are available and recommended as a tool to measure oral health outcomes in intervention studies dealing with several oral conditions [Mashoto et al., 2010; Kieffer et al., 2012; Abanto et al., 2013; Johal et al, 2014; Cantekin et al., 2014; de Paula et al., 2015; Turton et al., 2015; Yeh et al., 2016; de Souza et al., 2017]. Evidence for this recommendation should be appraised by reviewing the literature of intervention studies reporting the ability of different OHRQoL questionnaires to detect clinical changes over time, that is, the responsiveness of these instruments [Guyatt et al., 1989; Tsakos et al., 2010].

A previous systematic review reported the quality of evidence for changes in OHRQoL of children and adolescents following interventions such as oral rehabilitation in daily practice or under general anesthesia, orthodontic treatment, and treatment of traumatic dental injuries to be moderate [Antunes et al., 2013]. Recently, 2 other systematic reviews addressed the impact of caries treatment under general anesthesia on the OHRQoL of children and adolescents [Knapp et al., 2017; Park et al., 2018]. Knapp et al. [2017] observed a significant inconsistency in the quality of studies reporting changes by means of a variety of validated and nonvalidated OHRQoL questionnaires. Park et al. [2018] found short-term evidence of improved OHRQoL, with a large effect size. According to these authors, there is evidence to support the recommendation of oral rehabilitation under general anesthesia, based on strict indications, as a way to improve OHRQoL for young and uncooperative children with early childhood caries [Park et al., 2018].

However, there is a lack of information on the effect of caries intervention on OHRQoL in clinical settings and including different age groups. Therefore, this systematic review and meta-analysis were undertaken to assess the responsiveness of validated OHRQoL questionnaires to dental caries intervention in children, adolescents, and young adults.

\section{Materials and Methods}

A protocol was developed to answer the following PICO question: "Are validated OHRQoL questionnaires responsive to caries intervention procedures in children, adolescents and young adults?" Specific items included in the protocol were as follows:
- Population: systemically healthy children, adolescents, and young adults.

- Intervention: validated OHRQoL questionnaires applied before and after any dental caries intervention.

- Comparison or control:

- A group that did (not) receive basic caries intervention or a group that had no need for dental caries intervention.

- A single group pretest and posttest following caries intervention.

- Outcome: Responsiveness of OHRQoL questionnaires to intervention related to dental caries.

The protocol was registered at the international prospective register of systematic review, PROSPERO, under the identification number CRD42017080858 [National Institute for Health Research, 2017]. This systematic review followed the guidance from the PRISMA statement [Stovold et al., 2014].

\section{Eligibility Criteria}

Studies considered eligible for this review were randomized clinical trials (RCT), controlled clinical trials (CCT), and prospective case series (PCS), with no restriction for the follow-up period. These studies included children, adolescents, and young adults up to the age of 35 years who answered validated OHRQoL questionnaires before and after the intervention(s) for dental caries.

Intervention studies for oral conditions other than dental caries were excluded as well as studies in languages other than English, scientific publications in format of editorial, letter to editor, expert's opinion, and conference abstracts.

\section{Data Sources and Search Strategy}

The search strategy included any oral intervention study whose main outcome was the responsiveness of OHRQoL questionnaires. A search was conducted in August 2017 (updated in May 2018) by the first reviewer (N.R.A.). The search was carried out in 5 databases: Medline (via PubMed), Scopus, Cochrane, Livivo, and Web of Sciences. Google Scholar and Open Gray were assessed as additional literature. Few articles were recommended by experts.

The search strategy in Medline via PubMed was:

1 "Oral health quality of life questionnaire" OR "QoL questionnaire" OR questionnaire OR "oral health related quality of life" OR "OHRQoL" OR "surveys and questionnaires" (MeSH Terms) OR "surveys and questionnaires"

2 "Dental care" (MeSH Terms) OR "dental care" OR "oral care" OR “dental treatment” OR “oral health” (MeSH Terms) OR “oral health"

3 "Responsiveness" OR "longitudinal study" OR "longitudinal" OR "longitudinal evaluation" OR "prospective study" OR "clinical changes" OR "clinical study" OR "clinical trial” OR "dental intervention" OR "oral intervention"

4 “1 AND 2 AND 3"

The search strategies for different databases were adapted according to their individual requirements.

\section{Reliability, Study Selection, and Data Extraction}

Two independent reviewers (N.R.A., N.D.-T.) were calibrated for the study screening by 2 rounds of inclusion/exclusion of 30 randomly selected studies based on their titles and abstracts. After the first round, a nonweighted kappa value of 0.60 was achieved. 
After training sessions with intensive discussion of inclusion/exclusion criteria, the second round showed an absolute agreement between the 2 reviewers with an inter-reviewer nonweighted kappa value of 1.00 .

The selection process was performed in 2 phases. Phase- 1 was carried out in a web application specific for systematic reviews (Rayyan ${ }^{\circledR}$, Qatar Computing Research Institute), in which 2 blinded reviewers (N.R.A., N.D.-T.) screened the title and abstracts of all identified references. Any disagreements with regard to the eligibility criteria were mutually discussed and, if necessary, a third reviewer was involved (L.S.A.). In phase-2, the same 2 reviewers applied the eligibility criteria to full-text articles; if a consensus was not achieved, the third reviewer (L.S.A.) was consulted to make a final decision.

Relevant data of the 26 selected studies were independently extracted (N.R.A., N.D.-T.) and recorded in an Excel file designed for the study. Any disagreement was discussed as stated above, and experts on OHRQoL questionnaires (L.F.P.) and on caries (J.C.C.) data extractions were consulted in case of doubt. When the results of a given study were detailed in $>1$ paper, the most complete data set series was included. The data recorded were instrument applied, authors and year of publication, country in which the study was carried out, study design, setting, treatment modality, follow-up period, age, sample size, gender, outcomes, and conflict of interest. The authors were contacted if full-text articles were not obtained, or in case of missing information. The references were crosschecked to assure that all selected studies were included.

\section{Risk of Bias and Quality Assessment}

The quality assessment was also carried out independently (N.R.A., N.D.-T.). Disagreements were solved by discussion until a consensus was reached. The third reviewer (L.S.A.) was consulted to solve persistent discordance between the first and the second reviewer. For RCT, quality assessment was performed according to Higgins et al. [2003]. For CCT and PCS, risk of bias in non-randomized studies of interventions was used [Sterne et al., 2016].

\section{Data Analysis}

Mean values of the main outcome were directly pooled with weighted mean differences (WMDs) and 95\% CIs. Statistical heterogeneity was estimated by means of the chi-square test $(p<0.05)$ and I-square index $\left(I^{2}\right)$, which enabled to assess the magnitude of the inconsistency. Values of the $I^{2}$ over $50 \%$ were classified as high, $25-50 \%$ as moderate and $<25 \%$ as low [Higgins and Thompson, 2002]. The significance level was set at 0.05 . At the same time, the average difference of the investigated outcome was measured using standard technique on meta-analysis of random effect. Publication bias was initially reviewed by inspecting the graph Begg's Funnel Plot graphic. Subsequently, the Egger's test regression method with a significance level of $10 \%$ [Egger et al., 1997] was performed. Trim and Fill method identified the possible effects of the absence of studies with summary measures in the meta-analysis [Duval and Tweedie, 2000]. This was followed by a sensitivity analysis and regressions of subgroups to identify the source of heterogeneity of the studies included in the systematic review. Methodological heterogeneity was assessed in nonrandomized studies according to the risk of bias (low, moderate, and high) and according to mean (decayed, missing, and filled teeth, $\mathrm{dmft}$ ) scores.
In meta-regressions, the following covariates were used: risk of bias, study design, use of general anesthesia for dental intervention, mean dmft, and statistic relevance according to Galbraith's heterogeneity graphic [Dinnes et al., 2005]. Data analysis was performed in the STATA ${ }^{\circledR}$ statistical package 15 for Windows version, serial number: 301506206729.

\section{Level of the Evidence}

The Grading of Recommendations Assessment, Development, and Evaluation was used to assess the quality of the evidence and grading the strength of evidence of the included studies in the meta-analysis. The Grading of Recommendations Assessment, Development, and Evaluation approach to rating the quality of evidence considers the risk of bias, the inconsistency of results, the indirectness of evidence, the imprecision, and the publication bias as reasons to possibly rate down the quality of evidence. In contrast, the large magnitude effect, all plausible confounding, and the dose - response gradient may rate up the quality. The quality of assessment was rated into high, moderate, low, and very low level of evidence [Needleman et al., 2005]. The analysis of recommendation was based on the comparison between groups that received any intervention for dental caries with OHRQoL questionnaire (Early Childhood Oral Health Impact Scale, ECOHIS).

\section{Results}

\section{Study Selection}

From a total of 1,451 studies identified after removal of duplicates from different databases, 1,422 were excluded following the screening of titles and abstracts. Eight other studies were excluded after full-text reading, 2 of which included young adults up to the age of 35 years without age subgroup analysis.

Five further studies were included following crosschecking of the reference lists of all articles and expert decision, resulting in a total of 34 full-text articles assessed for eligibility. Finally, 26 studies were included in the qualitative analysis and 14 studies in the meta-analysis (Fig. 1). The reasons for exclusion of studies included in the full-text analysis are listed in Table 1.

\section{Descriptive Results}

Table 2 describes the characteristics of the selected studies. The majority of the studies were PCS with a single group pretest and posttest study design $(n=19)$. Five studies were CCT and only 2 were RCT. The studies were performed in 12 countries, being one-third conducted in hospital settings. The age of the studied subjects ranged from 3 to 19 years. The socioeconomic status (SES) of the subjects was only reported in 9 studies, 4 of which were included in the meta-analysis. The sample size considering both test and control groups varied 
Table 1. Selected studies excluded and reasons for exclusions $(n=8)$

\begin{tabular}{ll}
\hline Reasons for exclusion & Studies \\
\hline Full text not obtained $(n=2)$ & $\begin{array}{l}\text { Cunnion et al., } 2010 \\
\text { Filstrup et al., } 2003\end{array}$ \\
\hline Study without validated OHRQoL questionnaire $(n=1)$ & El Batawi et al., 2014 \\
\hline Studies based on the same data-base of other studies $(n=2)$ & Arrow and Klobas, 2016 \\
\hline Studies on OHRQoL and dental caries intervention, but without subgroup analysis & Thomson and Malden, 2011 \\
for patients under 35 years old $(n=2)$ & Hyde et al., 2006 \\
\hline Missing data for analysis $(n=1)$ & Yeh et al., 2016 \\
\hline
\end{tabular}

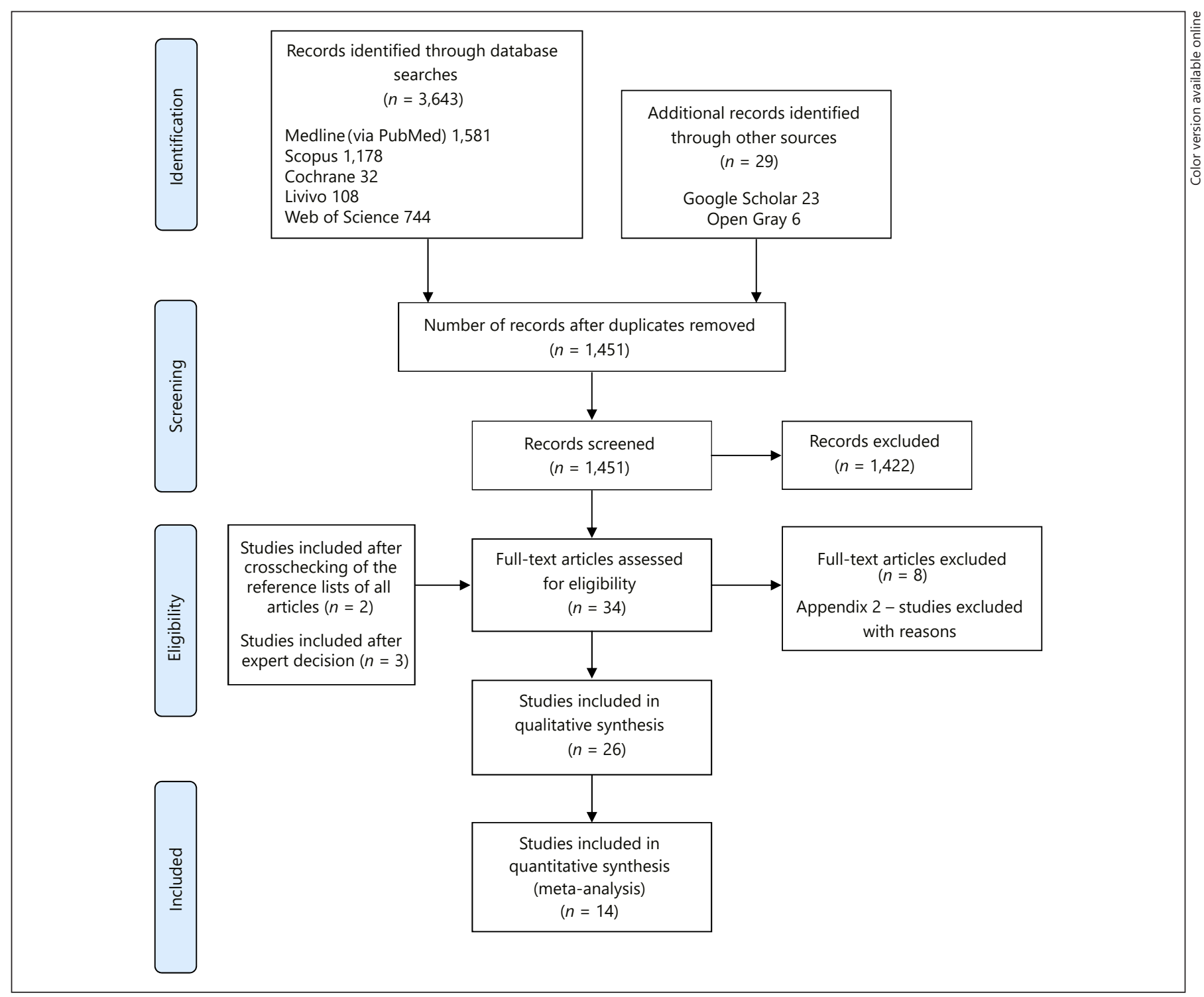

Fig. 1. Flow diagram for study selection according to PRISMA guidelines. 


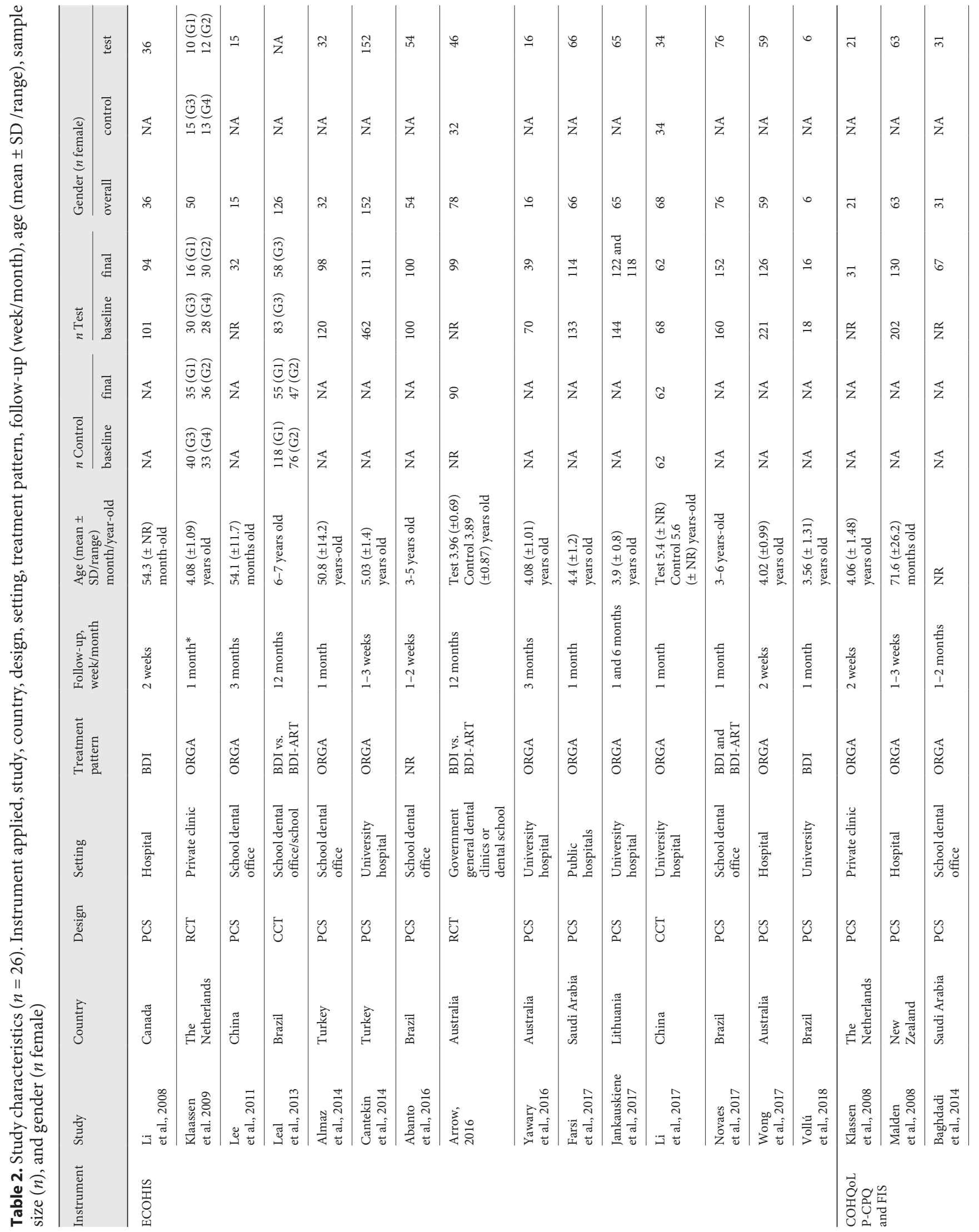




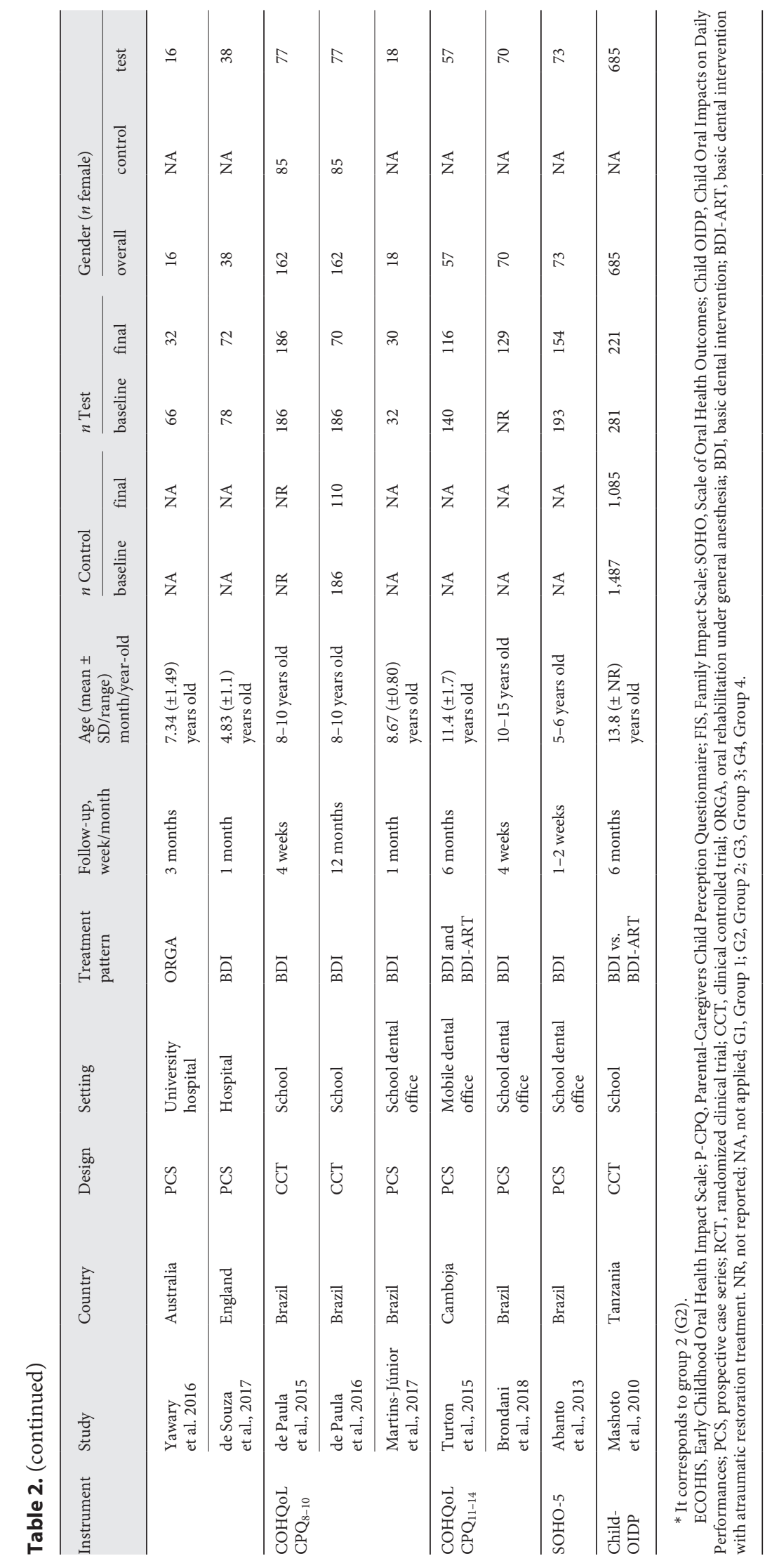


from 18 to 1,487 participants at baseline examination and from 16 to 221 at follow-up. The numbers of participants were 3,522 in the control group (baseline $=$ 2,002 ; final $=1,520$ ) and 5,917 in the test group (baseline $=3,102$; final $=2,815$ ).

A total of 6 instruments were used for the assessment of changes in OHRQoL in different studies as follows: ECOHIS (for 14 studies), Parental-Caregivers Child Perception Questionnaire and the Family Impact Scale (for 5 studies), Child Perception Questionnaire 8-10 (for 3 studies), Child Perception Questionnaire 11-14 (for 2 studies), Scale of Oral Health Outcomes for 5-year-old children (for 1 study), and Child Oral Impacts on Daily Performances (for 1 study). The Parental-Caregivers Child Perception Questionnaire and Family Impact Scale, the Child Perception Questionnaire $8-10$ as well as the Child Perception Questionnaire 11-14 are sub-types of the instrument Child Oral Health Quality of Life Questionnaire. Some studies used $>1$ instrument to assess OHRQoL.

Table 3 shows the study outcomes $(n=26)$ with mean control and test OHRQoL score, effect size, standardized response mean, and minimally importance difference. The primary outcome, changes on overall OHRQoL, was assessed during a follow-up period ranging from 1 week to 6 months.

Regarding caries data, the majority of the included studies used validated caries diagnostic classifications, namely, the World Health Organization criteria $(n=17)$ and the International Caries Detection Assessment System $(n=3)$, whereas 6 studies did not report the criteria used to record dental caries.

Caries experience was expressed as mean number of $\mathrm{dmft}$ (DMFT/dmft-index for permanent and primary dentition, respectively). The mean DMFT/dmft scores ranging from 0.6 to 8.0 (SD 3.7-1.7) and from 1.9 to 13.5 to (SD 2.1-3.1), respectively.

Dental caries intervention procedures were oral health instruction, prophylaxis, therapeutic fluoride, fissure sealant, fillings, stainless steel crown, pulpotomy, pulpectomy, and tooth extraction.

\section{Risk of Bias}

The quality assessment of the selected studies is summarized in Tables 4 and 5. A greater number of nonrandomized studies $(n=15)$ had low and moderate risk of bias than serious risk of bias $(n=9)$ according to the Risk of Bias in Non-randomized Studies of Intervention.

Quality of Life Questionnaires to Dental Caries Interventions

\section{Meta-Analysis}

The studies included in the meta-analysis were grouped under the ECOHIS instrument $(n=14)$. Figure 2 depicts the Forest plot of overall standard mean difference ECOHIS scores before and after dental caries treatment. The overall standardized WMDs of ECOHIS scores was -1.24 (95\% CI -1.68 to $-0.81, p<0.001)$, demonstrating an improvement in the OHRQoL. However, the heterogeneity was very high, with $I^{2}$ of $97 \%$ (95\% CI 95-97\%, $p<0.001)$.

Publication bias was measured using Egger regression and visual funnel plot inspection (Fig. 3). No publication bias was observed (Egger's test: $p=0.822$ ). This finding was confirmed by the trim-and-fill test, which showed that the number of publications and their expected association measurement were consistent with the estimate measured in the present systematic review. Galbraith graphic showed 6 studies as sources of heterogeneity.

Further subgroups analysis of the overall standard mean difference ECOHIS scores before and after dental treatment according to risk of bias showed significant difference within the RCT subgroup analysis, but with high heterogeneity $\left(-0.43,95 \% \mathrm{CI}-1.03\right.$ to $0.16 ; I^{2} 69.3 \%, p=$ $0.071)$. When the nonrandomized studies were considered, the subgroups had a WMD (95\% CI) as follows: low risk of bias -2.08 ( -3.77 to $\left.-0.40 ; I^{2} 98.0 \%, p<0.001\right)$, moderate risk of bias -1.54 ( -2.06 to $1.03 ; I^{2} 92.3 \%, p<$ $0.001)$, and serious risk of bias $-0.77\left(-1.55\right.$ to $0.02 ; I^{2}$ 95.9\%, $p<0.001)$.

The standard mean difference ECOHIS scores before and after dental treatment were $-1.46\left(-2.63\right.$ to $-0.29 ; I^{2}$ $97.6 \%, p<0.001)$ according to low and $-1.43(-2.69$ to $\left.-0.18 ; I^{2} 95.9 \%, p<0.001\right)$ according to high mean $\mathrm{dmft}$ scores.

The meta-regressions did not present study design ( $p=0.764)$, risk of bias $(p=0.870)$, treatment under general anesthesia $(p=0.460)$, and $\mathrm{dmft}(p=0.713)$ as possible causes of heterogeneity.

As a general trend, the quality of the evidence was very low and the strength of evidence was weak. The quality of evidence was not rated down by publication bias (0 points), whereas together the risk of bias, the indirectness of evidence, and the imprecision in addition to the inconsistency of results rated the quality down by 3 and 2 points, respectively. The quality of evidence was neither rated up the large magnitude effect nor by the dose - response gradient. Only the plausible confounding rated up the quality of the evidence by 1 point. 
Table 3. Study outcomes $(n=26)$ with mean control and test OHRQoL score, effect size (ES), standardized response mean (SRM), and minimally importance difference (MID)

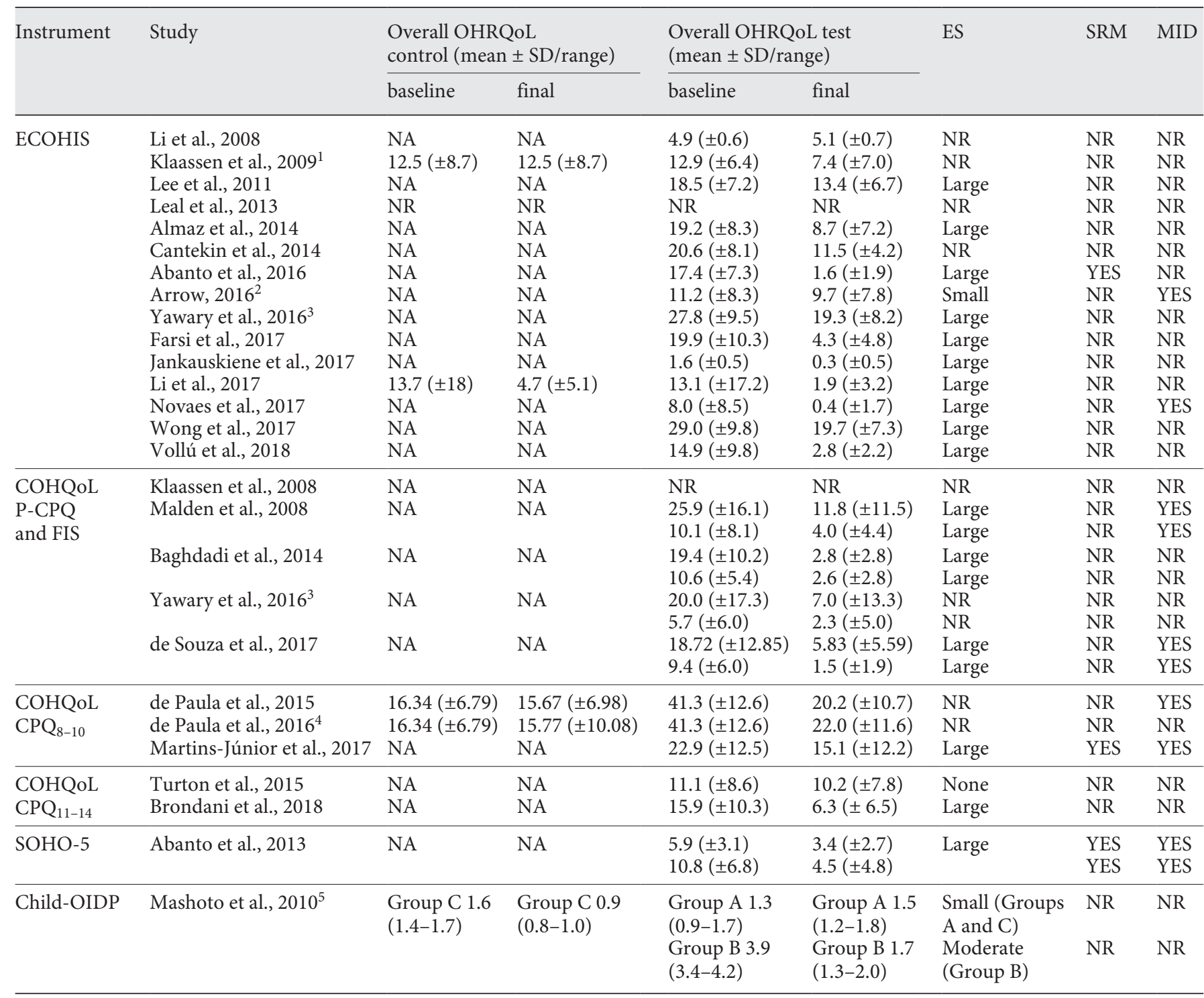

\footnotetext{
${ }^{1}$ It corresponds to G2 and G4.

2 The OHRQoL scores correspond to the mean value of test and control groups.

${ }^{3}$ Two instruments applied in the same study.

${ }^{4}$ Follow-up study de Paula et al., 2015.

${ }^{5} \mathrm{CI}$ reported.
}

NR, not reported; NA, not applicable; OHRQoL, Oral Health-Related Quality of Life; ES, effect size; SRM, standardized response mean; MID, minimally importance difference; ECOHIS; Early Childhood Oral Health Impact Scale; P-CPQ, Parental-Caregivers Child Perception Questionnaire; FIS, Family Impact Scale; SOHO, Scale of Oral Health Outcomes; Child-OIDP, Child Oral Impacts on Daily Performances; G2; group 2; G4, group 4. 
Table 4. Evaluation of the risk of bias of individual RCT $(n=2)$, as suggested by the Cochrane reviewers' handbook and by the CONSORT statement: study design, selection bias, performance bias, detection bias, attrition bias, reporting bias and other potential source of bias, categorized by OHRQoL instrument applied

\begin{tabular}{|c|c|c|c|c|c|c|c|c|c|c|c|}
\hline \multirow[t]{2}{*}{ Instrument } & \multirow[t]{2}{*}{ Reference } & \multicolumn{2}{|c|}{ Study design } & \multicolumn{2}{|c|}{ Selection bias } & \multirow{2}{*}{$\begin{array}{l}\text { Performance } \\
\text { bias blinding } \\
\text { of participants/ } \\
\text { personnel }\end{array}$} & \multirow{2}{*}{$\begin{array}{l}\text { Detection } \\
\text { bias Blinding } \\
\text { of outcome } \\
\text { assessment }\end{array}$} & \multirow{2}{*}{$\begin{array}{l}\text { Attrition bias } \\
\text { incomplete } \\
\text { data outcome }\end{array}$} & \multirow{2}{*}{$\begin{array}{l}\text { Reporting } \\
\text { bias }\end{array}$} & \multirow{2}{*}{$\begin{array}{l}\text { Other } \\
\text { potential } \\
\text { source of } \\
\text { bias funding }\end{array}$} & \multirow{2}{*}{$\begin{array}{l}\text { Overall } \\
\text { risk of } \\
\text { bias }\end{array}$} \\
\hline & & design & $\begin{array}{l}\text { design } \\
\text { (specific) }\end{array}$ & $\begin{array}{l}\text { sequence } \\
\text { generation }\end{array}$ & $\begin{array}{l}\text { allocation } \\
\text { concealment }\end{array}$ & & & & & & \\
\hline \multirow[t]{2}{*}{ ECOHIS } & $\begin{array}{l}\text { Klaassen } \\
\text { et al., } 2009\end{array}$ & RCT & Parallel & $\checkmark$ & $\checkmark$ & $\sqrt{ }$ & $?$ & $x$ & $\checkmark$ & Private & $x$ \\
\hline & $\begin{array}{l}\text { Arrow, } \\
2016\end{array}$ & RCT & Parallel & $?$ & $?$ & $?$ & $?$ & $x$ & $?$ & Public & $x$ \\
\hline
\end{tabular}

$\checkmark$, low risk of bias.

$\times$, high risk of bias.

?, unclear risk of bias.

RCT, randomized clinical trial; OHRQoL, oral health-related quality of life; ECOHIS, Early Childhood Oral Health Impact Scale.

No meta-analysis was performed for the remaining 12 studies included in the systematic review because they did not have sufficient data for analysis.

\section{Discussion}

The present systematic review and meta-analysis investigated the available evidence for improvement in OHRQoL of children and adolescents in young adults following caries intervention procedures. Almost all included studies, 24 out of 26 , showed significant reduction between the mean OHRQoL scores assessed before and after the caries intervention, indicating improvement in OHRQoL. However, the quality of the evidence was very low and its strength was weak.

It is important to bear in mind that the responsiveness of different instruments might be influenced by conditions such as selection of the population studied and its treatment needs. The study by Li et al. [2008] reported limited responsiveness to clinical changes of the ECOHIS instrument when applied to a population with low caries treatment needs. Such a result was likely as treatment needs of the population under study were already low at the beginning of the trial. Mashoto et al. [2010] using the Child-Oral Impacts on Daily Performance questionnaire found higher improvement in OHRQoL in a group of children receiving only oral health education compared to a group receiving ART restorative intervention. This finding suggests that either the observed improvement was related to other conditions than caries or that both groups had similar caries experience at the baseline.

Quality of Life Questionnaires to Dental Caries Interventions
In this systematic review, the positive effect of caries treatment in OHRQoL was further analyzed by the quality assessment of the selected studies, which indicated that more than half of the nonrandomized studies had either low risk of bias [Abanto et al., 2016; MartinsJúnior et al., 2018; Novaes et al., 2017] or moderate risk of bias [Klaassen et al., 2008; Mashoto et al., 2010; Lee et al., 2011; Cantekin et al., 2014, de Paula et al., 2015; Turton et al., 2015; Farsi et al., 2018; Jankauskiene et al., 2017; Li et al., 2017; de Souza et al., 2017; Brondani et al., 2018; Vollú et al., 2018]. Potential limitations of individual studies were the selection of participants into studies and missing data after caries intervention treatment which in their turn could have influenced changes on OHRQoL.

Equally, the quantitative effect of caries treatment on OHRQoL was assessed by means of a meta-analysis, which included studies using ECOHIS instrument. The Forest plot illustrates a significant improvement in the OHRQoL of children following caries treatment under general anesthesia (WMD $=-1.24 ; p<0.001)$. However, the heterogeneity was very high $\left(I^{2}=97 \%\right.$; $\left.p<0.001\right)$ in accordance with the findings reported by Knapp et al. [2017], but in contrast to those by Park et al. [2018] who reported a meta-analysis on the ECOHIS instrument with no evidence of heterogeneity. In the present meta-analysis, the subgroup analysis identified 6 studies as sources of heterogeneity [Li et al., 2008; Cantekin et al., 2014; Abanto et al., 2016; Arrow, 2016; Farsi et al., 2018; Jankauskiene et al., 2017].

Our results showed that the quality of evidence of the effect of caries interventions on OHRQoL was very low. Most studies were PCS, comparing the same subjects be- 


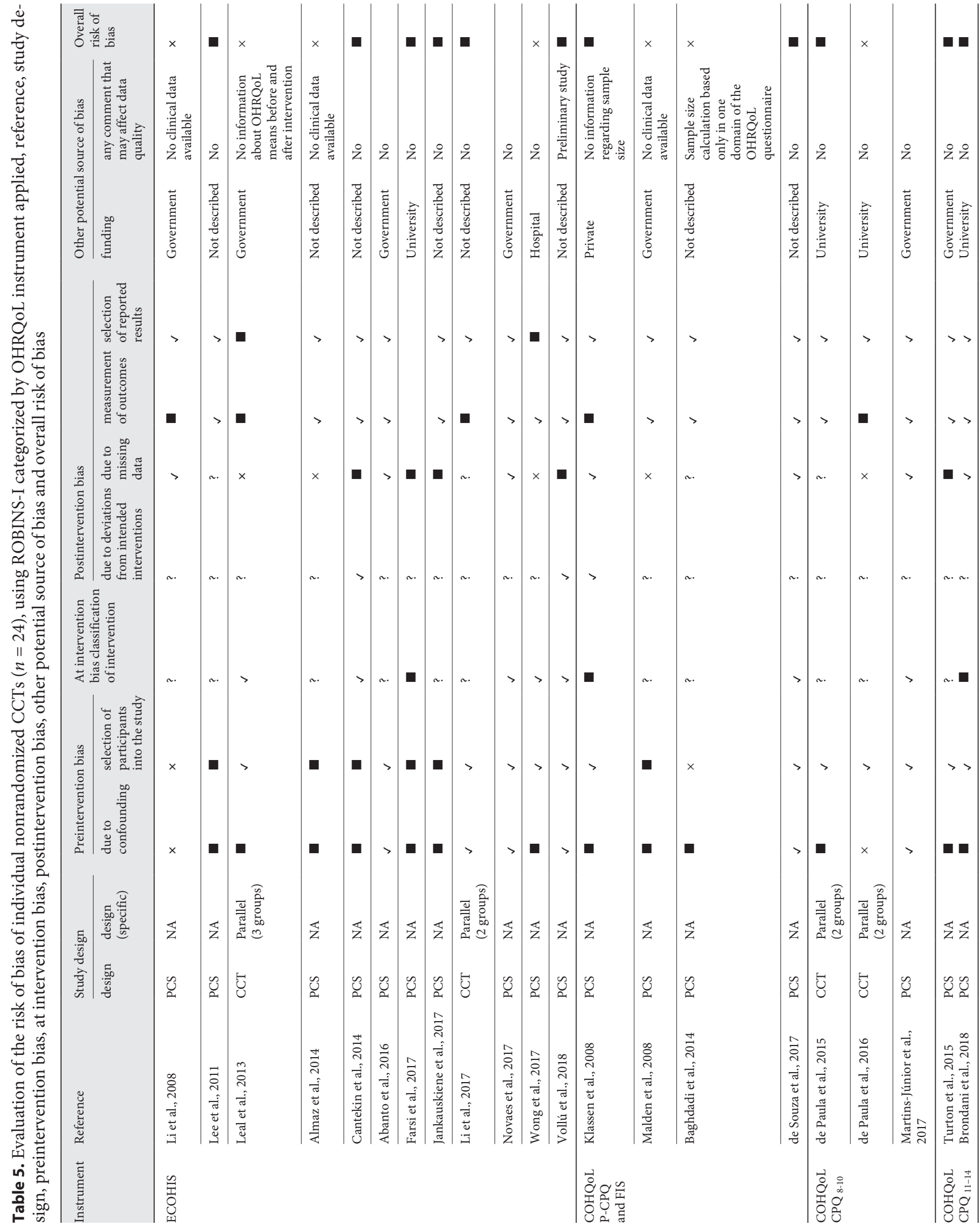




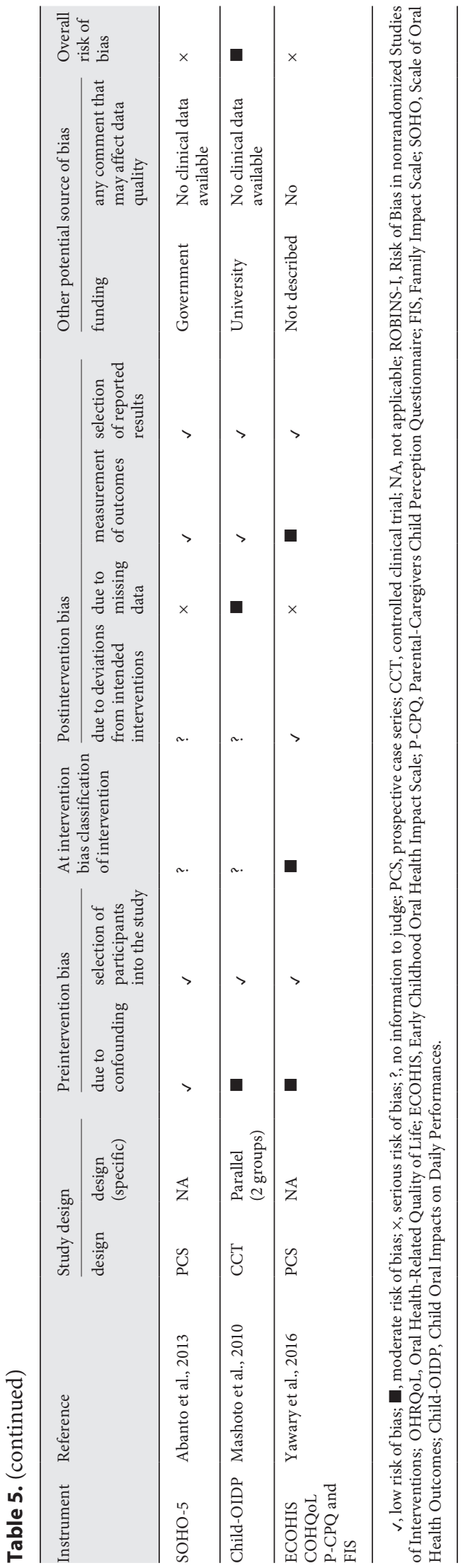

Quality of Life Questionnaires to Dental Caries Interventions fore/after treatment, without external comparison. This study design rated down the confidence that the estimated effect was correct. Nevertheless, one may acknowledge that PCS is the ideal study design to comply with ethical requirements relating to caries treatment in children, adolescents, and young adults, such as those included in the present systematic review. From the ethical perspective, it is not acceptable that patients in need of treatment for dental caries are not treated and included in a (non)-RCT as control group unless the patients/parents refuse the treatment. Although caries lesions should reach a certain level of severity to cause discomfort or pain, studies on improvement in OHRQoL following caries treatment are likely to report on treatment of moderate and severe caries lesions [Krisdapong et al., 2013; Aimée et al., 2017].

Only 2 RCT provided information regarding a true control group, but both studies were assessed as having high risk of bias [Arrow, 2016; Klassen et al., 2009]. Moreover, high heterogeneity and absence of studies analyzing the dose-response gradient were important factors responsible for the low quality of evidence.

One may argue that SES of patients would indirectly influence the outcome since low SES patients are likely to have high caries experience, which in its turn would impact negatively on OHRQoL. This assumption was not investigated in meta-analysis due to missing data on SES in the selected studies.

Even though the target population for the present systematic review included children, adolescents, and young adults, only one study on young adults with age subgroup of 15-19 years old [Mashoto et al., 2010] out of 3 initially selected studies [Hyde et al., 2006; Mashoto et al., 2010; Yeh et al., 2016] remained in the analysis. The reason for exclusion of the studies carried out by Hyde et al. [2006] and Yeh et al. [2016] was the absence of age subgroup analysis (Appendix 2). The responsiveness of OHRQoL questionnaires to caries interventions in young adults needs further investigation.

Within the limitations of this systematic review and meta-analysis, there is evidence that OHRQoL of children and adolescents improved following the treatment of dental caries and its consequences. The quality of the evidence of the effect of caries interventions on OHRQoL was very low and the strength of the evidence was weak. In spite of this, dental treatment for caries and its consequences is highly recommended as abstaining from treatment is likely to result in a deterioration of OHRQoL. However, further good quality research is warranted to strengthen the evidence. 


\begin{tabular}{|c|c|c|c|c|c|}
\hline Authors & Sample & $\operatorname{SMD}(95 \% \mathrm{Cl})$ & $\begin{array}{l}\% \\
\text { Weight }\end{array}$ & & \\
\hline Abanto et al., 2016 & 100 & $-2.95(-3.35,-2.55)$ & 7.16 & $\leftarrow-\quad \quad$ & \\
\hline Almaz et al., 2014 & 98 & $-1.35(-1.66,-1.04)$ & 7.34 & 1 & \\
\hline Arrow, 2016 & 99 & $-0.19(-0.46,0.09)$ & 7.39 & i & \\
\hline Cantekin et al., 2014 & 311 & $-1.41(-1.58,-1.23)$ & 7.53 & is & \\
\hline Farsi et al., 2017 & 114 & $-1.93(-2.25,-1.62)$ & 7.33 & $-\begin{array}{l}-\$ \\
1\end{array}$ & \\
\hline Jankauskiene et al., 2017 & 118 & $-2.59(-2.94,-224)$ & 7.27 & $-8-\quad 1$ & \\
\hline Klaassen et al., 2009 & 22 & $-0.81(-1.43,-0.19)$ & 6.61 & $\frac{1}{1}=-$ & \\
\hline Lee et al., 2011 & $\begin{array}{l}22 \\
32\end{array}$ & $-0.72(-1.23,-0.22)$ & 6.91 & $1-$ & \\
\hline Li L et al., 2017 & 32 & $-0.90(-1.27,-0.53)$ & 7.23 & $\frac{1}{i}-$ & \\
\hline Li L et al., 2017 & 62 & $0.31(0.02,0.59)$ & 7.38 & 1 & - \\
\hline Li S et al., 2008 & 94 & $-1.23(-1.48,-0.99)$ & 7.44 & $\frac{1}{-1}$ & \\
\hline Novaes et al., 2017 & 152 & $-1.66(-2.48,-0.81)$ & 6.00 & $\rightarrow-1$ & \\
\hline Vollu et al., 2018 & 16 & $-1.07(-1.34,-0.81)$ & 7.41 & $i^{-}$ & \\
\hline Wong et al., 2017 & 126 & $-0.96(-1.43,-0.49)$ & 7.00 & +- & \\
\hline Yawary et al., 2016 & 39 & $-1.24(-1.68,-0.81)$ & & & \\
\hline \multirow{4}{*}{$\begin{array}{l}\text { Overall }\left(I^{2}=96.0 \%, p=0.000\right) \\
R^{2}(95 \% \mathrm{Cl}): 95.0 \% ; 97.0 \%\end{array}$} & & & & i & \\
\hline & & & & I & \\
\hline & & & & -3.35 & 3.35 \\
\hline & & & & Favourable & Unfavourable \\
\hline
\end{tabular}

Fig. 2. Forest plot of overall SMD ECOHIS scores before and after dental treatment. SMD, standard mean difference.

Fig. 3. Funnel plot. SMD of individual ECOHIS studies (horizontal axis) plotted against SE (SMD) (vertical axis). SE, standard error; SMD, standard mean difference.

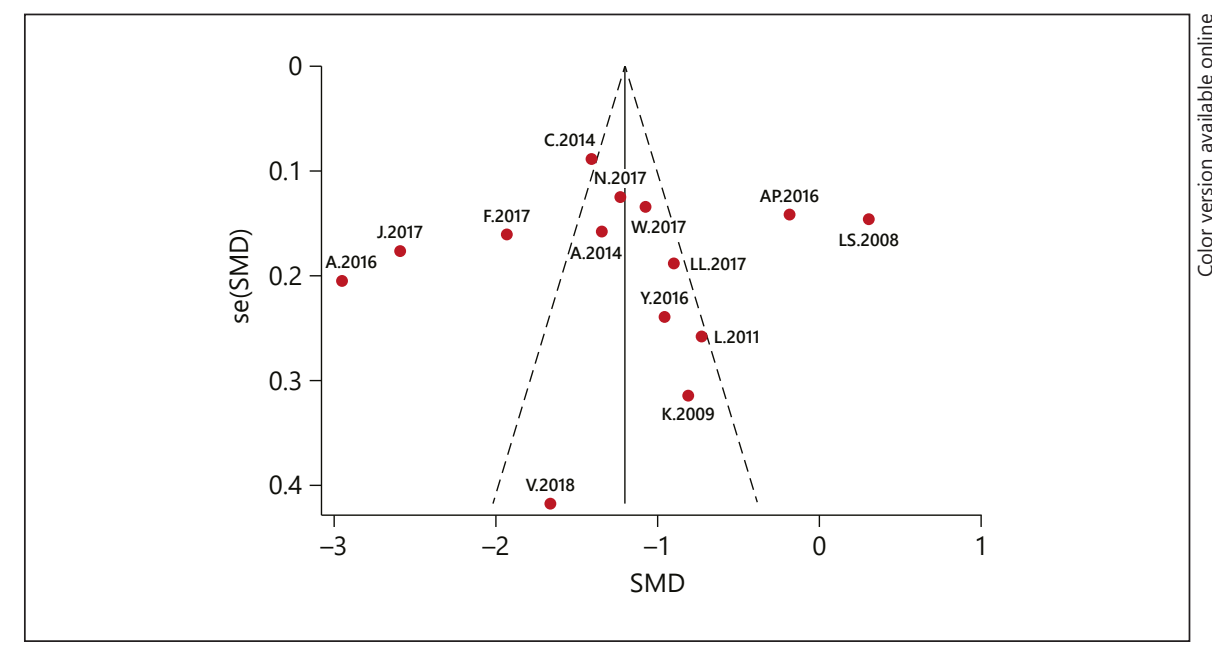

\section{Disclosure Statement}

The authors have no conflicts of interest to declare.

\section{Acknowledgments}

We would like to acknowledge all the contacted authors, who promptly answered to our requests and added valuable information for the present study. Additionally, we thank Professor Eliete Neves Guerra, University of Brasilia, Brazil, for her help in the electronic search and overall support.

\section{Authors Contribution}

N.R.A.: first reviewer, protocol design, data collection, manuscript. N.D.-T.: second reviewer, protocol design, data collection. L.S.A.: third reviewer, protocol design. G.Á.B.: protocol design, expertise on systematic reviews. L.F.P.: protocol design, expertise on OHRQoL questionnaires. H.D.M.: protocol design, manuscript, subcoordination. J.C.C.: protocol design, expertise on caries data, manuscript, coordination. All authors critically revised the manuscript. 


\section{References}

Abanto J, Paiva SM, Sheiham A, Tsakos G, Mendes FM, Cordeschi T, et al. Changes in preschool children's OHRQoL after treatment of dental caries: responsiveness of the B-ECOHIS. Int J Paediatr Dent. 2016 Jul; 26(4):259-65.

Abanto J, Tsakos G, Ardenghi TM, Paiva SM, Raggio DP, Sheiham A, et al. Responsiveness to change for the Brazilian Scale of Oral Health Outcomes for 5-year-old children (SOHO-5). Health Qual Life Outcomes. 2013 Aug;11(11):137.

Aimée NR, van Wijk AJ, Maltz M, Varjão MM, Mestrinho HD, Carvalho JC. Dental caries, fluorosis, oral health determinants, and quality of life in adolescents. Clin Oral Investig. 2017 Jun;21(5):1811-20.

Almaz ME, Sönmez IS, Oba AA, Alp S. Assessing changes in oral health-related quality of life following dental rehabilitation under general anesthesia. J Clin Pediatr Dent. 2014;38(3): 263-7.

Antunes LA, Andrade MR, Leão AT, Maia LC, Luiz RR. Systematic review: change in the quality of life of children and adolescents younger than 14 years old after oral health interventions: a systematic review. Pediatr Dent. 2013 Jan-Feb;35(1):37-42.

Arrow P, Klobas E. Child oral health-related quality of life and early childhood caries: a noninferiority randomized control trial. Aust Dent J. 2016 Jun;61(2):227-35.

Arrow P. Responsiveness and sensitivity of the Early Childhood Oral Health Impact Scale to primary dental care for early childhood caries. Community Dent Oral Epidemiol. 2016 Feb; 44(1):1-10.

Baghdadi ZD. Effects of dental rehabilitation under general anesthesia on children's oral health-related quality of life using proxy short versions of OHRQoL instruments. ScientificWorldJournal. 2014 Jan;2014:308439.

Brondani B, Emmanuelli B, Alves LS, Soares CJ, Ardenghi TM. The effect of dental treatment on oral health-related quality of life in adolescents. Clin Oral Investig. 2018 Jul;22(6):2291-7.

Cantekin K, Yildirim MD, Cantekin I. Assessing change in quality of life and dental anxiety in young children following dental rehabilitation under general anesthesia. Pediatr Dent. 2014 Jan-Feb;36(1):12E-7E

Cunnion DT, Spiro A 3rd, Jones JA, Rich SE, Papageorgiou $\mathrm{CP}$, Tate $\mathrm{A}$, et al. Pediatric oral health-related quality of life improvement after treatment of early childhood caries: a prospective multisite study. J Dent Child (Chic). 2010 Jan-Apr;77(1):4-11.

de Paula JS, Sarracini KL, Meneghim MC, Pereira AC, Ortega EM, Martins NS, et al. Longitudinal evaluation of the impact of dental caries treatment on oral health-related quality of life among schoolchildren. Eur J Oral Sci. 2015 Jun;123(3):173-8.

de Paula JS, Sarracini KL. Ambrosano GM, Pereira AC, Meneghim MC, Mialhe FL. Im- pact of a dental care program on the quality of life of children with and without caries. Braz Oral Res. 2016 Dec;30(1):e139.

de Souza MC, Harrison M, Marshman Z. Oral health-related quality of life following dental treatment under general anaesthesia for early childhood caries - a UK-based study. Int $\mathrm{J}$ Paediatr Dent. 2017 Jan;27(1):30-6.

Dinnes J, Deeks J, Kirby J, Roderick P. A methodological review of how heterogeneity has been examined in systematic reviews of diagnostic test accuracy. Health Technol Assess. 2005 Mar;9(12):1-113.

Duval S, Tweedie R. Trim and fill: A simple funnel-plot-based method of testing and adjusting for publication bias in meta-analysis. Biometrics. 2000 Jun;56(2):455-63.

Egger M, Davey Smith G, Schneider M, Minder C. Bias in meta-analysis detected by a simple, graphical test. BMJ. 1997 Sep;315(7109):62934.

El Batawi HY, Panigrahi P, Awad MA. Perceived outcomes and satisfaction of Saudi parents and their children following dental rehabilitation under general anesthesia: A 2-year follow-up. J Int Soc Prev Community Dent. 2014 Dec;4(3 Suppl 3):S153-60.

Farsi DJ, Farsi NJ, El-Housseiny AA, Damanhouri WH, Farsi NM. Responsiveness of the Arabic version of the ECOHIS to dental rehabilitation under general anaesthesia. Int J Paediatr Dent. 2018 Jan;28(1):52-61.

Filstrup SL, Briskie D, da Fonseca M, Lawrence L, Wandera A, Inglehart MR. Early childhood caries and quality of life: child and parent perspectives. Pediatr Dent. 2003 Sep-Oct;25(5): 431-40.

Guyatt GH, Deyo RA, Charlson M, Levine MN, Mitchell A. Responsiveness and validity in health status measurement: a clarification. J Clin Epidemiol. 1989;42(5):403-8.

Higgins JP, Thompson SG, Deeks JJ, Altman DG. Measuring inconsistency in meta-analyses. BMJ. 2003 Sep;327(7414):557-60.

Higgins JP, Thompson SG. Quantifying heterogeneity in a meta-analysis. Stat Med. 2002 Jun; 15;21(11):1539-38.

Hyde S, Satariano WA, Weintraub JA. Welfare dental intervention improves employment and quality of life. J Dent Res. 2006 Jan;85(1):79-84.

International prospective register of systematic reviews [Internet]. National Institute for Heath Research (NHS) [cited 2017 Dec 6]. Available from: https://www.crd.york.ac.uk/ PROSPERO/.

Jankauskiené B, Virtanen JI, Narbutaité J. Followup of children's oral health-related quality of life after dental general anaesthesia treatment. Acta Odontol Scand. 2017 May;75(4):255-61.

Johal A, Fleming PS, Al Jawad FA. A prospective longitudinal controlled assessment of pain experience and oral health-related quality of life in adolescents undergoing fixed appliance treatment. Orthod Craniofac Res. 2014 Aug; 17(3):178-86.
Kieffer JM, van Wijk AJ, Ho JP, Lindeboom JA. The internal responsiveness of the Oral Health Impact Profile-14 to detect differences in clinical parameters related to surgical third molar removal. Qual Life Res. 2012 Sep;21(7): 1241-7.

Klaassen MA, Veerkamp JS, Hoogstraten J. Dental treatment under general anaesthesia: the short-term change in young children's oralhealth-related quality of life. Eur Arch Paediatr Dent. 2008 Sep;9(3):130-7.

Klaassen MA, Veerkamp JS, Hoogstraten J. Young children's Oral Health-Related Quality of Life and dental fear after treatment under general anaesthesia: a randomized controlled trial. Eur J Oral Sci. 2009 Jun;117(3): 273-8.

Knapp R, Gilchrist F, Rodd HD, Marshman Z. Change in children's oral health-related quality of life following dental treatment under general anaesthesia for the management of dental caries: a systematic review. Int J Paediatr Dent. 2017 Jul;27(4):302-12.

Krisdapong S, Prasertsom P, Rattanarangsima K, Sheiham A. School absence due to toothache associated with sociodemographic factors, dental caries status, and oral health-related quality of life in 12 - and 15-year-old Thai children. J Public Health Dent. 2013;73(4):321-8.

Leal SC, Bronkhorst EM, Fan M, Frencken JE. Effect of different protocols for treating cavities in primary molars on the quality of life of children in Brazil-1 year follow-up. Int Dent J. 2013 Dec;63(6):329-35.

Lee GH, McGrath C, Yiu CK, King NM. Sensitivity and responsiveness of the Chinese ECOHIS to dental treatment under general anaesthesia. Community Dent Oral Epidemiol. 2011 Aug;39(4):372-7.

Li L, Wang H, Han X. Oral health-related quality of life in pediatric patients under general anesthesia: A prospective study. Medicine (Baltimore). 2017 Jan;96(2):e5596.

Li S, Malkinson S, Veronneau J, Allison PJ. Testing responsiveness to change for the early childhood oral health impact scale (ECOHIS). Community Dent Oral Epidemiol. 2008 Dec;36(6):542-8.

Malden PE, Thomson WM, Jokovic A, Locker D. Changes in parent-assessed oral health-related quality of life among young children following dental treatment under general anaesthetic. Community Dent Oral Epidemiol. 2008 Apr;36(2):108-17.

Martins-Júnior PA, Almeida L, Silva VS, Paiva SM, Marques LS, Ramos-Jorge ML. Sensitivity and responsiveness to change for the Brazilian version of the child perceptions questionnaire for 8 to 10 -year-old children. J Public Health. 2018;26(1):15-21.

Mashoto KO, Astrøm AN, Skeie MS, Masalu JR. Changes in the quality of life of Tanzanian school children after treatment interventions using the Child-OIDP. Eur J Oral Sci. 2010 Dec;118(6):626-34. 
Needleman I, Suvan J, Moles DR, Pimlott J. A systematic review of professional mechanical plaque removal for prevention of periodontal diseases. J Clin Periodontol. 2005;32(s6 Suppl 6):229-82.

Novaes TF, Pontes LR, Freitas JG, Acosta CP, Andrade KC, Guedes RS, et al.; CARDEC collaborative group. Responsiveness of the Early Childhood Oral Health Impact Scale (ECOHIS) is related to dental treatment complexity. Health Qual Life Outcomes. 2017 Sep; 15(1): 182 .

Park JS, Anthonappa RP, Yawary R, King NM, Martens LC. Oral health-related quality of life changes in children following dental treatment under general anaesthesia: a meta-analysis. Clin Oral Investig. 2018 Nov;22(8):2809-18.

Paula JS, Tôrres LH, Ambrosano GM, Mialhe FL. Association between oral health-related quality of life and atraumatic restorative treatment in school children: an exploratory study. Indian J Dent Res. 2012 Nov-Dec;23(6):738-41.

Severo Alves L, Dam-Teixeira N, Susin C, Maltz M. Association among quality of life, dental caries treatment and intraoral distribution in 12-year-old South Brazilian schoolchildren. Community Dent Oral Epidemiol. 2013 Feb; 41(1):22-9.

Sterne JA, Hernán MA, Reeves BC, Savović J, Berkman ND, Viswanathan M, et al. ROBINS-I: a tool for assessing risk of bias in nonrandomised studies of interventions. BMJ. 2016 Oct;355:i4919.

Stovold E, Beecher D, Foxlee R, Noel-Storr A. Study flow diagram in Cochrane systematic review updates: an adapted PRISMA flow diagram Systematic Reviews. Syst Rev. 2014 May; 3:54.

Thomson WM, Malden PE. Assessing change in the family impact of caries in young children after treatment under general anaesthesia. Acta Odontol Scand. 2011 Sep;69(5):257-62.

Tsakos G, Bernabé E, D'Aiuto F, Pikhart H, Tonetti M, Sheiham A, et al. Assessing the minimally important difference in the oral impact on daily performances index in patients treated for periodontitis. J Clin Periodontol. 2010 Oct;37(10):903-9.

Turton BJ, Thomson WM, Foster Page LA, Saub $\mathrm{R}$, Ishak AR. Responsiveness of the Child Per- ceptions Questionnaire11-14 for Cambodian children undergoing basic dental care. Int J Paediatr Dent. 2015 Jan;25(1):2-8.

Vollú AL, da Costa MD, Maia LC, Fonseca-Gonçalves A. Evaluation of Oral Health-Related Quality of Life to Assess Dental Treatment in Preschool Children with Early Childhood Caries: A Preliminary Study. J Clin Pediatr Dent. 2018;42(1):37-44.

Wong S, Anthonappa RP, Ekambaram M, McGrath C, King NM, Winters JC. Quality of life changes in children following emergency dental extractions under general anaesthesia. Int J Paediatr Dent. 2017 Mar;27(2): 80-6.

Yawary R, Anthonappa RP, Ekambaram M, McGrath C, King NM. Changes in the oral health-related quality of life in children following comprehensive oral rehabilitation under general anaesthesia. Int J Paediatr Dent. 2016 Sep;26(5):322-9.

Yeh DY, Kuo HC, Yang YH, Ho PS. The Responsiveness of Patients' Quality of Life to Dental Caries Treatment-A Prospective Study. PLoS One. 2016 Oct;11(10):e0164707. 